Revue scientifique sur la conception et l'aménagement de l'espace

\title{
Le jardin, lieu esthétique d'un (dés)ordre humain : la naturalisation du politique et du littéraire chez Claude Simon et Patrick Chamoiseau
}

The Garden, The Aesthetic Locus of a Human (Dis)Order : The Naturalisation of Policy and Literature in the Works of Claude Simon and Patrick Chamoiseau

\section{Hannes De Vriese}

\section{(2) OpenEdition}

\section{Journals}

Édition électronique

URL : http://journals.openedition.org/paysage/8033

DOI : 10.4000/paysage.8033

ISSN : 1969-6124

Éditeur :

École nationale supérieure du paysage de Versailles-Marseille, Institut national des sciences appliquées Centre Val de Loire - École de la nature et du paysage, École nationale supérieure d'architecture et de paysage de Bordeaux, École nationale supérieure d'architecture et de paysage de Lille, Agrocampus Angers

\section{Référence électronique}

Hannes De Vriese, «Le jardin, lieu esthétique d'un (dés)ordre humain : la naturalisation du politique et du littéraire chez Claude Simon et Patrick Chamoiseau », Projets de paysage [En ligne], 14 | 2016, mis en ligne le 22 août 2016, consulté le 30 juillet 2020. URL : http://journals.openedition.org/paysage/ 8033 ; DOI : https://doi.org/10.4000/paysage.8033

Ce document a été généré automatiquement le 30 juillet 2020

Projets de paysage 


\title{
Le jardin, lieu esthétique d'un (dés)ordre humain : la naturalisation $\mathrm{du}$ politique et $\mathrm{du}$ littéraire chez Claude Simon et Patrick Chamoiseau
}

\author{
The Garden, The Aesthetic Locus of a Human (Dis)Order : The Naturalisation of \\ Policy and Literature in the Works of Claude Simon and Patrick Chamoiseau
}

\section{Hannes De Vriese}

Deux hommes se retirent dans un jardin après une vie de combat. Le premier, Lacombe Saint-Michel, est un personnage des Géorgiques de Claude Simon. Général révolutionnaire, il rejoint, après une carrière passée à se battre aux quatre coins de l'Europe, le domaine familial dont il assume depuis des années la gestion par correspondance. Le second, Balthazar Bodule-Jules, personnage principal dans Biblique des derniers gestes de Patrick Chamoiseau, est aussi un combattant. Après s'être engagé dans des luttes de décolonisation partout dans le monde, il rentre dans la commune de Saint-Joseph pour terminer sa vie dans son jardin.

2 Ces personnages d'épopée choisissent de mourir dans un havre de paix, un hortus conclusus qui les retire des remous du siècle et qui leur permet de retrouver un calme pacifique. Le jardin se donne ainsi à lire comme un espace édénique, comme «le dernier repaire qui s'offre [...] à l'homme qui rêve d'échapper au cauchemar de l'histoire et aux espaces inhabitables de la modernité » (Précy, 2011, p. 63ํ). Il promeut la possibilité d'un ressourcement, d'une réconciliation avec un soi ballotté par les affres de l'histoire, car ces héros sont les instruments de récits dislocateurs de leur intégrité, qui les bousculent, les dispersent jusque dans leur identité, leur nom (cf. la réduction du patronyme à des initiales, LSM et Bibiji) et la démultiplication du moi dans la prolifération des alter ego ${ }^{2}$. 
Il est important toutefois de distinguer le jardin humain de l'espace édénique. Seul ce dernier constitue un réel refuge, contrairement au premier qui, par le travail d'aménagement et d'entretien qu'il demande, renvoie l'homme à la vita activa (Harrison, 2010, p. 19). Or les jardins dans lesquels se retirent les protagonistes ont bien été créés par la main de l'homme: Lacombe Saint-Michel prend sa retraite dans le domaine dont il a hérité et qu'il a entretenu lui-même alors que Bodule-Jules, plutôt que de chercher refuge dans la forêt sauvage de sa mère adoptive Man L'Oubliée, choisit de remettre en état « un ancien jardin de sa maman Manotte et de son papa Limorelle » (Chamoiseau, 2003, p. 765).

4 Les jardins représentés dans les textes doivent ainsi moins être envisagés comme des espaces en huis clos édénique que comme des lieux de branchement avec le monde. Ils s'apparentent à ce que Michel Foucault nomme des hétérotopies ${ }^{3}$, dont la marginalité réflexive permet de les situer en dehors de l'espace du monde, en même temps que dans une double stratégie d'écho et de critique: ils constituent de la sorte une « contestation à la fois mythique et réelle de l'espace où nous vivons » (Foucault, 2001, p. 756). Le jardin n'est pas hermétique à l'histoire, mais se constitue en écho du politique, soit en tant que reproduction en miniature d'une organisation de la société, soit en tant que lieu de négation du système politique existant. Dans l'un et l'autre cas, le jardin constitue la métaphore spatiale et naturelle d'une présence au monde.

5 C'est précisément cette naturalisation de l'être-au-monde à travers le jardin que nous nous proposons d'étudier et qui constitue, à notre sens, un point de rencontre entre Patrick Chamoiseau et Claude Simon. Non pourrons à cet égard non seulement prendre appui sur Les Géorgiques et Biblique des derniers gestes, mais également sur Le Jardin des Plantes, texte dans lequel Claude Simon donne à voir un jardin planétaire à travers des vues aériennes, ou Texaco, roman dans lequel Patrick Chamoiseau met à l'honneur l'énergie débordante que dégage un quartier populaire. Ces œuvres affichent clairement un sentiment de contemporanéité au monde, qui permet de l'apprécier comme une triple entité : d'abord une planète "parmi une infinité d'autres », ensuite l'écoumène ou "cette Terre-là " que nous appelons nôtre et enfin "notre Monde ", cette planète bleue que l'entreprise spatiale a mise en évidence (Lussault, 2013, p. 18-21). En tant que lieu de branchement avec le monde, l'espace du jardin renvoie plus encore à « la dimension relationnelle de l'existence » (Bourriaud, 1998, p. 64). Il s'agit proprement d'un espace interstitiel qui, «tout en s'insérant plus ou moins harmonieusement et ouvertement dans le système global, suggère d'autres possibilités d'échanges que celles qui sont en vigueur dans ce système " (Bourriaud, 1998, p. 14). Le traitement littéraire de l'espace du jardin serait alors susceptible de mettre en évidence "l'impromptu ardent du monde" (Glissant, 1997, p. 114) selon une logique de la «pensée archipélique» et du «chaos-monde » chers à Édouard Glissant. Le motif littéraire du jardin remplit en d'autres mots une fonction triple: il se fait le reflet critique d'une politique de l'espace, il rend compte de la conscience contemporaine d'un monde multiple et il renvoie à de nouvelles procédures d'interaction avec cette complexité-là. 


\section{Les Géorgiques ou l'échec d'une naturalisation du politique}

Dans Les Géorgiques, LSM appelle bien de ses vœux un hortus conclusus où il pourra terminer sa vie: «J'ai constamment été dans les armées actives depuis quarante ans, mais aujourd'hui [...] la retraite battra bientôt pour moi ; j'irai dans mon vieux château passer mes derniers jours et je pourrai encore jeter un regard en arrière sans regrets ; j'en jette aujourd'hui un bien agréable en me rappelant avec toi les moments que nous avons passés ensemble dans notre jeunesse : [...] le triomphe de quelque villageoise ou de quelque religieuse bornait notre ambition, heureux âge ! (Simon, 2013, p. 691.)

7 Le domaine autour du château recèle les promesses d'un possible « âge heureux » où les efforts et les peines de la vie politique et militaire s'estomperaient au profit d'une innocence retrouvée. En réalité, la retraite de LSM est avant tout marquée par une morne solitude et par la dégénérescence : le personnage n'est plus qu'un « tas de vieux os et de chair " (ibid., p. 892). Le jardin s'est transformé en lieu de désenchantement pour le général déchu. En effet, LSM, même assis sur la terrasse de son château, ne se départit ni de «sa vieille houppelande de soldat» (ibid., p. 895), ni de ses habitudes de militaire.

8 L'aménagement du jardin matérialise les nécessités d'un geste fortificateur, quadrillant une nature clôturée : le texte fait alterner les lettres de LSM à l'intendante Batti avec ses missives de guerre, soulignant par là la proximité entre l'homme de guerre et le jardinier (voir ibid., p. 677-678 et p. 681). La correspondance de LSM comporte à la fois un "précis d'agriculture " (ibid., p. 939) et un art de la guerre. Le général adopte d'ailleurs dans tous ses écrits un même style injonctif, qui trahit l'empiétement du militaire sur le pastoral :

«J'ai le projet de faire construire une terrasse au-dessus de l'enclos du coq: vous ferez planter des treilles le long de la muraille qui descend à la rivière, vous taillerez l'allée des ormeaux, vous visiterez exactement les deux allées de treilles qui aboutissent au Cabinet des lauriers, vous remplacerez ceux qui manquent, vous visiterez tous les prés de la rivière, vous remplacerez les saules et les peupliers qui manqueront, vous regarnirez tout le rivage du tombeau, vous regarnirez les peupliers de l'ovale de la petite fontaine, vous. » (Ibid., p. 677.)

9 La logique du paysagiste que l'on aperçoit ici ressemble en tout point à celui du stratège qui aménage des outils défensifs autour d'une place forte. La terrasse surplombe le jardin, les murailles sont doublées de murs végétaux que supportent les treilles. Les vides dans les rangs d'arbres doivent être comblés. Le domaine répond ainsi à une exigence de rendement, aussi bien sur un plan défensif que commercial. Empreints d'un prosaïsme pragmatique, les rapports de LSM à son jardin sont biaisés par la schématisation cartographique du lieu paysager. Le domaine demeure consigné dans l'abstraction des plans et la virtualité des projets :

«Il n'avait fait que traverser sans s'arrêter ce paysage, ces champs, ces collines, ce vallon, ces halliers qui pendant toutes ces années n'avaient existé pour lui que comme des choses tout à fait réelles, sans autre matière que l'immatérielle mémoire, abstraitement figurées sur ce plan qu'il emportait partout avec lui.» (Ibid., p. 893.)

Lorsque le général observe du haut de la terrasse la nature vivante qui l'entoure, il n'est pas en mesure de rentrer en contact avec celle-ci car il est « séparé de ce monde aux multiples frémissements, aux minuscules échos, par une vitre, une de ces épaisses 
plaques de verre » (ibid., p. 894). Une telle incapacité à se rendre compte de la réalité du domaine n'est pas seulement imputable à la vieillesse du personnage; elle manifeste le raide conditionnement des grilles de lecture que les cartes projettent sur le territoire et dont LSM reste prisonnier. Cette grille érige le domaine en emblème de la qualité sociale de son propriétaire. C'est une œuvre de prestige, à l'instar du buste censé représenter la force et la gloire de LSM. Jardin et œuvre sculpturale puisent dans les mêmes codes artistiques néoclassiques ${ }^{4}$, qui visent à figer la puissance du personnage dans une esthétique atemporelle. Les jardins du château répondent ainsi à une naturalisation du politique que Michel Conan met en évidence dans les jardins aristocratiques anglais de la fin du xvIII ${ }^{e}$ siècle :

«[...] l'art du jardin anglais [...] fut aussi un appareil idéologique du capitalisme anglais du xVIII siècle. Domaines et jardins se sont de plus en plus confondus, adoptant la forme de paysages arcadiens variés à la fois utiles et symboliques, de telle sorte que les jardins paysagers, au lieu de contenir des signes de l'engagement politique, sont devenus eux-mêmes l'expression d'une politique. Or, comme cette expression se confond formellement avec la nature et qu'elle est censée être la source de tout bien pour l'homme, la politique s'y est trouvée naturalisée, mise hors la critique, et hors l'histoire. » (Conan, 2004, p. 383.)

11 Les plantations en ligne droite ou "en quinconce » (Simon, 2013, p. 681) ne calquent pas seulement une disposition militaire, elles donnent aussi littéralement corps à un ordre social et politique immuable.

Si une telle conception politique, qui transforme le paysage en Arcadie immuable, convient mal aux convictions révolutionnaires de LSM, elle est en outre fallacieuse et fragile, puisque la propriété ne tardera pas à péricliter, comme en témoigne le narrateur :

«Entre les deux tours décapitées il ne subsiste plus de la terrasse qu'un faible

renflement de terrain aménagé en potager et cerné par les orties. » (Ibid., p. 661.)

LSM est après sa mort trahi par son propre jardin : les tours et la terrasse, attributs de la puissance du maitre, sont tombées en ruine, le potager et le verger sont à l'abandon, en signe de l'impuissance du géomètre de la nature dont le protagoniste a voulu se donner le statut.

Claude Simon décrit ainsi avec un regard à la fois fasciné et critique la vie et les œuvres de son ancêtre, le général Lacombe Saint-Michel ${ }^{5}$. Le jardin de celui-ci apparaît comme un lieu de pouvoir strictement hiérarchisé. De tels endroits verticaux figurent aussi dans les fictions de Patrick Chamoiseau : les formes géométriques régissent de même certains paysages, notamment celui de la ville dont « [les] rues [sont] toutes droites et se [coupent] carrées » (Chamoiseau, 2011, p. 213) et celui des plantations, entièrement organisées en cercles concentriques autour de la demeure du maitre. Il va de soi que la modernité urbaine aussi bien que la division de l'espace selon le système plantationnaire répondent d'une même volonté de maîtrise et de figement des espaces sociaux et naturels. Cependant, Patrick Chamoiseau, qui fustige la permanence d'une «domination devenue silencieuse » (Chamoiseau, 2002, p. 18), ne peut adopter le même regard que Claude Simon. L'auteur antillais décrit peu les espaces du pouvoir ${ }^{6}$ et préfère mettre en scène des espaces interstitiels qui s'opposent à l'ordre politique établi et qui œuvrent ainsi à la construction d'une identité non plus redevable à une généalogie racinaire mais à une narration aux prises avec le monde. 


\section{Biblique des derniers gestes et l'énergie rebelle des plantes}

14 Loin des grands domaines aux lignes strictes, les jardins que représente Patrick Chamoiseau se caractérisent en effet comme des lopins de terre, des «minuscule[s] jardin[s] ( (Chamoiseau, 2003, p. 34) privés, à la végétation exubérante et dont les soins reviennent à un individu ou une famille. La relation à la terre y est directe ; elle se passe de l'abstraction des cartes et laisse place à une jouissance toute poétique de la nature. Ainsi Balthazar Bodule-Jules est-il, contrairement à LSM, en mesure de contempler le paysage à partir de la terrasse de son jardin :

« De sa terrasse, dans le vidé des colibris ivres de sucre et de vie sédentaire, [...] on découvrait la campagne de Saint-Joseph, ses brumes flottantes, ses ombres vertes, et l'émergence céleste des pitons du Carbet que le vieil homme contemplait sans jamais se lasser. C'était pour lui un signe de la beauté mais surtout un bel espace de liberté où les nègres du temps de l'esclavage réfugiaient d'orgueilleuses résistances et l'inmontrable du désespoir. » (Ibid., p. 34-35.)

$15 \mathrm{Au}$ moment où le « vieux rebelle » vit retiré dans son jardin, ce ne sont plus les pitons et les mornes qui font figure d'espaces de liberté, mais c'est le jardin lui-même qui cristallise désormais la fulgurance de la résistance politique. Car le jardin, dans la mesure où il permet à Bibiji de "soutenir un siège de cent cinquante-cinq ans " (ibid., p. 37), se révèle aussi politisé et militarisé que chez Claude Simon. À travers le potager notamment, le vieil homme réalise un rêve d'autarcie :

"L'inépuisable rebelle avait maintenu cette autonomie alimentaire. Ce rapport ancestral à la tourbe nourricière se constituait pour lui en fondement des libertés pérennes. » (Ibid.)

16 La consommation de légumes du jardin apparaît chez Chamoiseau comme un outil de résistance. Il s'agit d'un moyen de survie pour les esclaves des habitations, les « hectares de cannes et de café » (ibid., p. 62) n'offrant pas de nourriture. De même, les parents de Marie-Sophie Laborieux créent leur jardin dans les mornes afin de subsister après l'abolition de l'esclavage ${ }^{7}$. À une époque davantage contemporaine, l'importance du jardin nourricier comme levier de liberté et d'indépendance s'avère d'autant plus essentielle :

«L'En-ville s'était développé ; à mesure-à mesure, il avait distendu son rapport à la

terre, supprimé ses jardins. » (Ibid., p. 284.)

Les légumes du jardin permettent de s'affranchir d'un approvisionnement extérieur et évitent d'être à la merci d'un maître ou d'un importateur.

17 Cependant, l'intention révolutionnaire qui sous-tend l'entretien du jardin dépasse sa seule capacité de vivier alimentaire, elle réside aussi en la force vitale des plantes mêmes. Dans le jardin, la végétation exhibe sa capacité de croissance. Ainsi, en désignant Texaco comme une « mangrove urbaine » (ibid., p. 336), l'urbaniste signifie la capacité de croissance naturelle de ce quartier populaire construit en marge de la ville lors de l'exode rural en Martinique. Dans une belle analyse du motif de la mangrove, Dominique Chancé constate que de Texaco à Biblique des derniers gestes, cette mangrove, envahie par des dealers, s'est transformée en lieu de désenchantement. Elle estime qu' « aucune structure n'est plus idéalisée dans l'ultime poétique de Biblique des derniers gestes; tout lieu est le site d'un enchantement possible et d'une inquiétude mélancolique, le site d'une ambivalence totale » (Chancé, 2011, p. 56). 
Il nous semble toutefois que le jardin de Bibiji se substitue à la métaphore végétale de la mangrove et continue de la sorte, à une échelle individuelle, l'entreprise identitaire et politique décrite dans Texaco. Ce jardin célèbre, tout comme la mangrove urbaine, la force de croissance sauvage des végétaux, mais il met aussi en scène une conscience apaisée, qui sait reconnaître "ses illusions » et qui accepte que les plantes comme les hommes ne soient que «le jouet du vivant» (Chamoiseau, 2003, p. 816). L'agressivité qu'ils portent en eux est un ennemi intérieur, "vaincre en soi la violence apaise le monde " (Chancé, 2011, p. 56). À partir de là, le jardin apparaît comme un lieu où l'entreprise de Texaco peut se réinventer; les forces individuelles s'aimantent pour construire un vivre-ensemble. Les gestes du jardinier Bibiji prolongent à cet égard, dans un champ d'action privé, les théories de l'urbaniste de Texaco:

\footnotetext{
« [Bibiji] ne recherchait plus l'ordre équitable des beautés domestiques, mais l'autoorganisation des énergies, l'équilibre négocié des puissances. Il raisonnait telle ou telle liane. Il contenait telle branche avec son sécateur qui ne le quittait plus. Il découchait des rhizomes agressifs, orientait leur horde... " (Chamoiseau, 2003, p. 816.)
}

Plutôt que de répondre à l'exécution d'un ordre programmé, le jardinier répond à une urgence situationnelle qui relève du kairos. L'ancien combattant a ainsi délaissé les armes pour une nouvelle forme de résistance, végétale cette fois. Il s'entoure désormais d'« orchidées très rares» (ibid., p. 34) et s'est créé un "cocon végétal» (ibid.) qui surprend ses visiteurs, davantage habitués à le voir en guerrier :

« On s'attendait à visiter un arsenal de guerres anciennes et de vestiges de champs de bataille, et non pas ces ciselures végétales dont il s'était fait une passion indévoilée et délicate autant. » (Ibid.)

Aux luttes armées s'oppose, par l'efflorescence d'un vivant raffiné, une résistance qui s'appuie sur la croissance des plantes et plus précisément sur une plante, l'orchidée, en mesure de symboliser l'identité locale. L'œuvre de Patrick Chamoiseau donne ainsi à voir, à travers la représentation du jardin, la naturalisation d'une rébellion politique. Celle-ci est de nature dynamique; elle s'oppose aux forces qui figent l'ordre de la société. L'extrait de Biblique des derniers gestes, en faisant état des " ciselures végétales », maintient le jardin dans le domaine des armes. Le sécateur, dans la main du jardinier, n'est pas tant un outil pour couper la plante, qu'un moyen de décupler la recrudescence de la végétation qui pourra à son tour hachurer, brouiller les formes rectilignes de l'espace urbain.

\section{La naturalisation de l'esthétique chez Claude Simon et Patrick Chamoiseau, de l'hortus conclusus au jardin planétaire}

Le terme "ciselures", renvoyant à l'art de la sculpture et à l'orfèvrerie, désigne en outre le jardin comme un objet d'art fabriqué par la main de l'homme, "surgi sola humana arte " (Blumenberg, 2010, p. 43), à l'aide du sécateur qui devient ici l'outil de l'artiste. Objet créé à partir d'un matériau végétal qui fait figure d'élément de nature " toujours-déjà-là ", selon les mots de Hans Blumenberg, le jardin peut être considéré comme "le dépassement de "l'imitation de la nature" " pour devenir une "préfiguration de la nature " (ibid., p. 89). Le jardin ne fait pas office d'ekhprasis de la nature, il est en réalité un objet hybride où la distinction entre nature et culture 
s'estompe ${ }^{8}$. Ainsi, le passage de la mangrove urbaine dans Texaco au jardin dans Biblique des derniers gestes démontre le cheminement d'une réflexion sur la distinction entre le culturel et le naturel et signale le soupçon que Patrick Chamoiseau porte sur l'imitation de la nature par les ouvrages de l'homme.

Le jardin allie une envie d'ordre humain à la poussée turbulente du végétal. Gilles Clément, dans un chapitre intitulé "Illusion de l'ordre illusion du désordre ", considère le jardin comme le lieu par excellence où le désordre sauvage et l'ordre humain coexistent et se neutralisent :

«C'est au jardin - au jardin seulement - que la nature est donnée à lire suivant un ordre particulier. Ailleurs, dans le paysage agricole, la nature est contredite de façon radicale. Et si le paysage n'est pas agricole on dit qu'il est sauvage, ce qui exclut la notion d'ordre. » (Clément, 2007, p. 19-20.)

Or, Patrick Chamoiseau et Claude Simon, en procédant, respectivement dans Biblique des derniers gestes et Le Jardin des plantes, à la description horticole, ouvrent l'objet littéraire au contingent du végétal et associent le geste scriptural au kairos du jardinier. Il y a là une naturalisation de l'esthétique littéraire. Celle-ci implique, dans les textes, un travail de sape de l'hortus conclusus et l'ouverture du texte au monde qui épouse le modèle du jardin planétaire. De ce point de vue, il est compréhensible que les textes éprouvent le besoin de défaire le dispositif de l'hortus conclusus et de faire éclater ses motifs géométriques. Les murs du jardin clos s'apparentent au cadre d'un tableau; ils enferment et étouffent la vie végétale ; ils ordonnent à l'excès.

Les textes simoniens offrent une vision radicale de l'hortus conclusus, estampillant le jardin clos comme un lieu mortifère qui s'associe à la vanité baroque. Ainsi, Lacombe Saint-Michel commande de clôturer la tombe de sa première épouse à l'aide d'arbres :

«Dans la division bleue où se trouve la tombe il commande de faire planter des peupliers d'Italie, des hêtres, des frênes et surtout des robiniers. » (Simon, 2013, p. 691.)

Il construit ainsi, au sein de son domaine, une section parfaitement étanche, représentée par la « division bleue » sur le plan, installée en guise d'écrin protecteur du souvenir de la femme qui peut ainsi demeurer intact, comme embaumé. Seulement, cet effort de conservation est voué à l'échec :

«Il ne subsiste de la tombe qu'une pierre rectangulaire parmi un entrelacs de ronces, feuilletée par les gels d'hiver et où l'inscription à moitié recouverte par des lichens est difficilement lisible. (Ibid., p. 669.)

Le monument en lui-même ne résiste pas aux affres du temps; pas plus que la morte « flottant mollement dans un liquide noir, putride, la longue et imputrescible chevelure ondulant, se déroulant en paresseuses volutes autour de la face aux orbites vides, à la bouche sans langue, sans lèvres, aux incisives saillantes sous le nez dévoré » (ibid., p. 895). L'ekphrasis du jardin, avec ses compartiments dédiés à la mémoire d'une morte, devient ici vanité, symbole de sa mort.

La dimension mortuaire du jardin clos n'est pas une exception dans l'œuvre simonienne. Le jardin familial près de Perpignan est d'abord le lieu où agonise la mère du narrateur. De même, dans une des dernières scènes du Jardin des plantes, le narrateur raconte dans un souvenir d'enfance comment il a failli se noyer dans un «bassin tapissé d'une litière de feuilles mortes rousses brunes rouille prune pourries gluantes » (Simon, 2006, p. 1167). 

vie aux formes géométriques, «aux pelouses et aux plates-bandes rectangulaires encadrées par les longues murailles rectilignes de platanes taillés chaque année au cordeau » (ibid., p.1158). Par la disposition du texte en îlots qui bousculent la circulation dans l'œuvre au gré d'une structure organique, le texte simonien réussit à " excéder la "ligne" " (Baetens, 2001, p. 31). L'œuvre s'inspire ainsi de la créativité débordante que met en abyme la « luxuriante végétation du jardin » de Picasso (Simon, 2006, p. 1168). Elle prend forme sur le « grand acacia » (ibid., p. 1073) qui pousse dans le jardin où la mère du narrateur a agonisé, arbre qui, par sa haute futaie et la mobilité de ses feuilles déborde le cadre du jardin (voir à ce sujet Clément-Perrier, 1998, p. 10 sqq.). La configuration arborescente et vivante de l'œuvre remédie à la mort de la mère. Dans le jardin du narrateur, les ombres des «feuilles s'entrecroisent en minces triangles noirs mouvants» (Simon, 2006, p.939), introduisant ce même jeu mobile de la végétation qui déconstruit l'hortus conclusus.

Quant à Biblique des derniers gestes, le récit déplie la vie tout en soubresauts de Bibiji selon une structure organique qui «fonctionne moins comme une arborescence que comme un rhizome» (Cornille, 2014, p. 99). L'ordonnancement des événements signifierait un reniement de la vie. Il convient alors, dans le jardin comme dans le texte, de « laisser ce frémissement, [de] ne plus voir sa menace » (Chamoiseau, 2003, 826). Ici encore la végétation brouille les lignes, elle se constitue en "marée de fleurs » qui « commenc[e] à vaincre les alignements de son jardin de survie » (ibid., p. 814).

La géométrie des œuvres littéraires est donc troublée par une poussée végétale; les clôtures du jardin et du texte vacillent, ouvrent un espace autrement fermé au monde. La mise à mal de la forme rectiligne autorise l'entrée en résonance d'un espace intérieur avec le monde extérieur. Le jardin littéraire devient, chez Claude Simon et Patrick Chamoiseau, un "jardin planétaire», selon l'expression de Gilles Clément. Certes, le jardinier-auteur respecte la poussée turbulente des plantes, mais il dispose aussi d'une liberté de configuration. C'est lui qui négocie la cohabitation des espèces et des expériences car le jardin « accueille le meilleur de tout, le meilleur des arbres et des fleurs, le meilleur des fruits et des lumières, le meilleur du "paysage" » (Clément, 2007, p. 118).

On comprend mieux à cet égard la citation de Montaigne que Claude Simon inscrit en exergue du Jardin des Plantes: il s'agit de représenter dans l'œuvre «les lopins » qui façonnent une expérience humaine. De son côté, le vieux Balthazar Bodule-Jules fait lui aussi le décompte de ses expériences pour les projeter dans son jardin :

«Il rameuta ses expériences passées, toutes ces divagations à travers la planète, ces sensations multiples, ces rencontres insensées, il les accepta, il les planta en lui, les déposa dans le terreau de son enfance comme des plantes enracinées pourtant prêtes à fleurir. (Chamoiseau, 2003, p. 813.)

La vie de Bibiji, ses voyages et expériences imprègnent la facture du jardin tout autant que l'œuvre littéraire qui retranscrit son itinéraire chaotique. Les textes assemblent ainsi les lieux visités par le jardinier-voyageur dans un ensemble qui redistribue la mappemonde en jardin planétaire vivant et personnel.

Chez Claude Simon aussi bien que chez Patrick Chamoiseau, l'esthétique littéraire, en s'inspirant de la forme et de l'énergie végétales, signifie un nouveau rapport au monde. En dépit d'une telle démarche commune, les auteurs, qui publient tous deux pendant les années 1990 mais qui appartiennent à des générations et à des aires culturelles

Projets de paysage, 14 | 2016 
différentes, se distinguent par la vision alternative qu'ils proposent. En effet, si tous deux critiquent la linéarité d'un rapport hiérarchique à l'espace, Claude Simon ne remet pas réellement en cause la pertinence des «systèmes vertébrés " (Appadurai, 2007, p. 39), c'est-à-dire de ces systèmes qui, comme les puissances coloniales et les États nations, s'appuient sur une structure verticale à visée coordonnatrice et régulatrice (ibid., p. 46). La forme arborescente qui marque son écriture est à cet égard significative: elle inscrit le cosmopolitisme planétaire du Jardin des plantes dans un mouvement démultiplié et pacifique qui contrebalance l'action guerrière de LSM dans Les Géorgiques. Claude Simon déplace certes le regard de la racine et du tronc vers la multiplicité des branches et la mobilité des feuilles, mais l'arbre n'en relève pas moins d'une conception vertébrée du monde, conception qu'Édouard Glissant décrit par l'image de la racine (Glissant, 1996, p. 59 sqq.). L'écriture de Patrick Chamoiseau répond, quant à elle, pleinement à une logique rhizomatique qui confère au quartier de Texaco et au jardin de Balthazar Bodule-Jules les caractéristiques de "l'ethnoscape ", terme par lequel Arjun Appadurai désigne de nouveaux lieux où des groupes « reconstruisent leur histoire et reconfigurent leur projet ethnique » (Appadurai, 2015, p. 91). Le projet littéraire de Patrick Chamoiseau répond ainsi bien mieux à la "poétique du divers " qu'Édouard Glissant appelle de ses vœux (Glissant, 1996). jardin donne à voir la capacité du texte à se constituer en archive vivante. Comme le dit Jacques Isolery :

"L'écriture trouve son équilibre dans une mise en corrélation spatio-temporelle des archives qui [...] désintègre la notion de structure interprétative dans une superstructure (dé-) concertante, infiniment ouverte par la reproductibilité de l'archive au cœur des multiples espaces de la représentation littéraire » (Isolery, 2005, p. 96.)

Le jardin planétaire ne présente donc pas une interprétation totalisante de l'expérience du monde, mais une juxtaposition significative des parcours. Après avoir passé sa vie à parcourir le monde, Bibiji peut ainsi affirmer que "ces errances avaient du sens" (Chamoiseau, 2003, p. 813), affirme Bibiji. Lui qui autrefois contemplait anxieusement la mappemonde, en « déplaçant son doigt sur la petite planète » (ibid., p. 543), sans pour autant pouvoir atteindre les lieux désignés, peut désormais accepter le (dés) ordre du monde. À travers le jardin, il réalise le conseil de Sarah-Anaïs-Alicia : «Pour vraiment vivre dans un de ces Lieux, il faut être partout...» (Ibid., p. 597.) Le jardin littéraire donne ainsi corps à un rêve d'ubiquité, il réconcilie l'homme avec le (dés) ordre dans un mouvement de synergie qui n'a rien d'une résignation.

Projets de paysage, 14 | 2016 


\section{BIBLIOGRAPHIE}

Appadurai, A., Après le colonialisme. Les conséquences culturelles de la globalisation (1996), traduit de l'anglais par F. Bouillot, Paris, Payot et Rivages, coll. « Petite Bibliothèque Payot », 2015.

Appadurai, A., Géographie de la colère. La violence à l'âge de la globalisation (2006), traduit de l'anglais pas F. Bouillot, Paris, Payot et Rivages, 2007.

Baetens, J., « Éloge de la ligne » dans Houppermans, S. (dir.), Crin. Claude Simon et Le Jardin des plantes, Amsterdam-New York, 2001, n 39, p. 31-42.

Bourriaud, N., L'Esthétique relationnelle, Dijon, Les Presses du Réel, coll. « Documents sur l'art », 1998.

Blumenberg, H., L'Imitation de la nature et autres essais esthétiques, traduit de l'allemand par I. Kalinowski et M. de Launay, Paris, Hermann, coll. « Le Bel Aujourd'hui », 2010.

Chamoiseau, P., Texaco (1992), Paris, Gallimard, coll. « Folio », 2011.

Chamoiseau, P., Biblique des derniers gestes (2002), Paris, Gallimard, coll. « Folio », 2003.

Chancé, C., « Patrick Chamoiseau, De La "mangrove urbaine" de Texaco à la "mangrove immonde" de Biblique des derniers gestes ", Ponti/Ponts, n 11, Milan, 2011, p. 41-59.

Clément, G., Le Jardin en mouvement. De la vallée au jardin planétaire (1994), Paris, Sens \& Tonka, 2007.

Clément-Perrier, A., Claude Simon. La fabrique du jardin, Paris, Nathan, coll. « 128 », 1998.

Conan, M., Essais de poétique des jardins, Florence, Leo S. Olschki, coll. « Gardini e paese », 2004, p. 383.

Cornille, J.-L., Chamoiseau... fils, Paris, Hermann, coll. « Fictions pensantes », 2014.

Foucault, M., « Des espaces autres » dans Defert, D., Ewald, F. et Lagrange, J. (dir.), Dits et Écrits, Paris, Gallimard, coll. « Quarto », 2001, p. 752-762.

Glissant, E., Traité du Tout-Monde. Poétique IV, Paris, Gallimard, 1997.

Glissant, E., Introduction à une poétique du divers, Paris, Gallimard, 1996.

Harrison, R. P., Jardins. Réflexions sur la condition humaine (2007), traduit de l'anglais par Fl.

Naugrette, Paris, Le Pommier, 2010.

Isolery, J., « Le jardin des traces dans les romans de Claude Simon » dans Sarkonak, R. (dir.), La Revue des Lettres modernes. Claude Simon 4. Le (dé) goût de l'archive, Paris, Minard, 2005, p. 57-99.

Lussault, M., L'Avènement du monde. Essai sur l'habitation humaine de la Terre, Paris, Seuil, coll. « La couleur des idées ", 2013.

Milne, L., Patrick Chamoiseau. Espaces d'une écriture antillaise, Amsterdam-New York, Rodopi, 2006.

Précy, J. de, Le Jardin perdu, traduit de l'anglais par M. Martella, Arles, Actes Sud, 2011.

Simon, C., Les Géorgiques (1981), CEuvres, textes présentés A. B. Duncan, Paris, Gallimard, « Bibliothèque de la Pléiade », 2013, vol. 2, p. 647-959.

Simon, C., Le Jardin des Plantes (1997), Euvres, textes présentés par A. B. Duncan, Paris, Gallimard, «Bibliothèque de la Pléiade », 2006, vol. 1, p. 945-1178. 


\section{NOTES}

1. Il est à noter que tout porte à croire que Jorn de Crécy, qui aurait publié cet essai sur le jardin en 1912, est un personnage de l'invention du traducteur.

2. On pense au frère de LSM et aux noms doubles récurrents chez les personnages de Biblique des derniers gestes.

3. Le jardin figure parmi les exemples d'hétérotopies (Foucault, 2001, p. 758-759).

4. Tout comme l'œuvre picturale décrite dans le prologue.

5. Le personnage de LSM renvoie au général Lacombe Saint-Michel. La famille de Claude Simon a découvert les archives de cet ancêtre par hasard et l'écrivain les exploite dans son roman.

6. Ni Biblique des derniers gestes, ni Texaco ne donnent réellement à voir cette " "matrice" stérile » qu'est l'habitation (voir Milne, 2006, p. 58).

7. Voir à cet égard «Le Noutéka des mornes", chant de guerre qui célèbre les capacités nourricières des jardins des mornes (Chamoiseau, 2011, p. 161-173).

8. Le jardin donnerait alors à voir, si l'on suit la réflexion de Hans Blumenberg, un état possible de la nature.

\section{RÉSUMÉS}

Cette réflexion examine le motif littéraire du jardin, en prenant appui sur les œuvres de Claude Simon (Les Géorgiques, Le Jardin des plantes) et de Patrick Chamoiseau (Texaco, Biblique des derniers gestes). Significatif d'un rapport particulier au monde, le jardin amène à prendre en compte des points communs entre les deux auteurs, mais demande aussi à resituer les textes dans un contexte de production particulier. L'œuvre simonienne porte la trace de l'écriture du nouveau roman; les textes antillais s'inscrivent dans un contexte postcolonial. Dans la littérature contemporaine, le motif du jardin n'induit pas l'évocation d'un idéal pastoral immobile. Au contraire, le jardin apparaît comme un lieu dynamique qui déstabilise l'ordonnancement préconçu par le jardinier, représentant alors un désordre vivant qui déboute l'homme de ses rôles de créateur unique et de destinataire privilégié de l'espace créé. Le jardinier doit négocier un équilibre; il ne se poste pas en collectionneur de plantes à aménager, mais en figure de l'artiste œuvrant à une « esthétique relationnelle " (Bourriaud, 1998). De même, l'auteur, en se référant au jardin, œuvre selon une poétique de l'interaction. Le jardin apparaît in fine comme un levier privilégié pour une «naturalisation de l'esthétique " littéraire, génératrice d'un biopatrimoine qui n'est pas simple ekphrasis résultant d'une représentation programmée, mais l'exécution d'un geste s'effectuant dans l'urgence situationnelle du kairos. L'espace aménagé répond dans ce cas toujours à un souhait d'équilibre; seulement celui-ci ne relève pas d'une perspective d'ordonnancement et de catégorisation des espèces, mais bien d'une fabrique végétale dynamique, d'un plein biologique qui paysage l'œuvre littéraire en mouvement.

This study examines the literary motif of the garden in the works of Claude Simon (Les Géorgiques, Le Jardin des Plantes) and Patrick Chamoiseau (Texaco, Biblique des derniers gestes). Representative of a special relationship to the world, the garden reveals commonalities between the authors, however, it also requires replacing the texts within a specific context. The Simonian oeuvre bears the traces of the nouveau roman; the texts from the Antilles are expressive of a post-colonial context. In contemporary literature the motif of the garden does not induce the evocation of an 
immutable pastoral ideal. On the contrary, the garden appears as a dynamic locus which destabilises the pre-designed arrangement of the gardener, thus representing a living disorder which dismisses man from his role as the sole creator and privileged beneficiary of the space created. The gardener must negotiate a balance; he does not present himself as a collector of plants to be arranged, but as an artist intent on achieving "relational aesthetics" (Bourriaud, 1998). In the same way, the author, by referring to the garden, is working with the poetics of interaction. The garden appears in fine as the preferred lever for "naturalising" a literary aesthetic at the inception of a bio-heritage which is not simply the ekphrasis resulting from a planned representation but the execution of a gesture in the situational urgency of the kairos. The developed space always addresses the need for an equilibrium; except that in this case it does not stem from an ordering and categorisation of species, but from a dynamic plant production, a biological profusion which landscapes the literary work in movement.

\section{INDEX}

Keywords : Contemporary literature, garden, Claude Simon, Patrick Chamoiseau, aesthetic, poetic

Mots-clés : littérature contemporaine, jardin, Claude Simon, Patrick Chamoiseau, esthétique, poétique

\section{AUTEUR}

\section{HANNES DE VRIESE}

Docteur en lettres modernes, Ater à l'université de Toulouse-Jean Jaurès, ses recherches portent sur l'écopoétique et l'écriture de la nature dans la littérature contemporaine, notamment sur la représentation de l'espace et du paysage, dans une perspective européenne et caribéenne. hannesdevriese[at]gmail[dot]com 